\title{
Study of Conventional and Novel Risk Factors of Ischemic Stroke in Eastern India
}

\author{
NIRENDRA M BISWAS, ${ }^{1}$ DIPENDRA N GHOSH, ${ }^{2}$ AMITABHA SENGUPTA, ${ }^{3}$ SUMIT SEN, ${ }^{4}$ DEBRAJ JASH,${ }^{5}$ KAUSHIK \\ SAHA, ${ }^{6}$ ARPITA BANERJEE, ${ }^{7}$ PRANAB MANDAL ${ }^{8}$
}

\begin{abstract}
:
Background: Stroke accounts as one of the leading cause of morbidity and mortality and third most common cause of death worldwide. In a significant percentage of stroke patients no definite cause can be identified. These group of unknown cause, the so-called 'cryptogenic strokes' are associated with some novel risk factors and now a major focus for clinical discussion.

Objectives: To find out conventional risk factors behind ischemic stroke and to establish correlation between ischemic stroke with elevated lipoprotein(a), Homocysteinemia, elevated High-sensitivity C-reactive protein (hs CRP),Systemic Lupus Erythematous (SLE) and chronic H.pylori infection and carotid intimo-medial thickness(CIMT).

Materials and methods: Thirty (30) admitted ischemic stroke patients based on WHO definition and confirmed by axial CT and or MRI Scan. were included. Thirty (30) controls were recruited from close relatives of the patients attending the Medicine OPD without any prior history of stroke or coronary heart diseases. Risk factors were documented on the basis of biochemical tests performed.

Results : Statistically significant association of ischaemic stroke with diabetes, hypertension, smoking, homocysteine, $L p(a)$, hsCRP, LVH, CIMT,H.pylori infection. No association of valvular heart disease, atrial fibrillation, A.N.A was found with ischaemic stroke.

Conclusion: In addition to measures that include blood sugar, blood pressure and lipid control routine estimation of $L p(a)$, homocysteine level, monitoring of atherosclerotic changes in carotid artery by Doppler study, echocardiographic monitoring of LVH should be done so that appropriate steps can be taken to avert an attack of stroke. Lastly early detection and eradication of H.pylori is always advisable to reduce the incidence of ischemic stroke.
\end{abstract}

Keywords: Ischaemic stroke, risk factors, correlation, diabetes mellitus, H.Pylori infection

\section{Introduction}

Stroke is one of the leading cause of morbidity and mortality and third most common cause of death worldwide standing next to coronary heart disease (CHD) and cancers. ${ }^{1,2}$

1. Associate professor, Department of General Medicine, NRS Medical College and Hospital

2. Post graduate trainee, Department of General Medicine, NRS Medical College and Hospital

3. Associate Professor, Dept of pulmonary Medicine, NRS Medical College and Hospital

4. Associate Professor, Dept of Dermatology, IPGMER,Kolkata

5. Post Graduate (MD) Trainee, Department of Pulmonary Medicine, NRS Medical College and Hospital

6. Assistant Professor, Department of Pulmonary Medicine, Burdwan Medical College and Hospital

7. R.M.O cum Clinical tutor,Department of Pulmonary Medicine, Calcutta Medical College

8. Associate Professor, Department of Pulmonary Medicine, N.R.S.Medical College \& Hospital

Correspondence : Dr. Debraj Jash, Post Graduate (MD) Trainee, Department of Pulmonary Medicine NRS Medical College and Hospital e mail-jashdebraj@ gmail.com
However unlike the Caucasians, Asians have a lower rate of CHD and a higher prevalence of stroke. ${ }^{[2,3]}$ World Health Organisation(WHO) defined stroke as 'rapidly developed clinical signs of focal disturbance of cerebral functions, lasting for more than 24 hours or leading to death, with no apparent cause other than vascular origin'. The disturbance of cerebral function is caused by three morphological abnormalities namely stenosis, occlusion and rupture of the arteries.

Risk factors associated for ischemic stroke are broadly classified into three groups. First group includes the preventable risk factors like smoking, lack of physical activities, unhealthy diet and obesity. Second group factors are treatable factors like hypertension, diabetes mellitus, dyslipidaemias, renal function impairment and emboli from diseased heart. Third group left with fixed risk factors like history of stroke or coronary heart disease in first degree relative in young age, early menopause, age, use of oral contraceptive pill etc.

In spite of these 25 to $39 \%$ of stroke patients no definite cause can be identified. These group of unknown cause, the socalled 'cryptogenic strokes' are associated with some novel risk factors and now a major focus for clinical discussion. 


\section{Objective of this study were}

i) To find out conventional risk factors behind ischemic stroke.

ii) Correlation between ischemic stroke with elevated lipoprotein (a), Homocysteinemia, and elevated Highsensitivity C-reactive protein (hs CRP).

iii) Correlation between ischemic stroke with Systemic Lupus Erythematous (SLE) and Anti Phospholipid Antibody (APLA) Syndrome.

iv) Correlation between ischemic stroke with chronic Helicobactor pylori (H.pylori) infection and

v) Association of stroke with carotid intimo-medial thickness.

\section{Material And Methods:}

\section{Place of Study:}

This study was conducted at NRS Medical College and Hospital, Kolkata, India in the department of Medicine from the period of February,2010 to December,2011. All patients provided informed consent and study protocol was approved by the Ethical committee of the Institution.

\section{Study Subject:}

Thirty (30) ischemic stroke patients who were admitted in Medicine Department were included. WHO definition of stroke was used for diagnosis. Confirmation of stroke was done by axial CT and or MRI Scan.

\section{Exclusion Criteria:}

i) Age less than 15 years

ii) Prior history of Stroke

iii) Haemorrhagic stroke following head injury

iv) Critically ill patients

\section{Control:}

Thirty (30) controls were recruited from Medicine Department. They included close relatives of the patients attending the Medicine OPD not having any prior history of stroke or coronary heart diseases.

Data Collection:

i) Complete Haemogram

ii) Blood sugar fasting, post prandial and $\mathrm{HbA}_{1} \mathrm{C}$ level.

iii) Serum urea and creatinine level

iv) Fasting lipid profile.

v) Serum LP (a) level

vi) ANA by indirect immunofluroscence method with hep2 cell.

vii) Serum Homocysteine level

viii) ECG and Echocardiography

ix) Carotid Doppler study for intimo medial thickness.

x) Upper GI Endoscopy with rapid urease test for H. Pylori infection.
Risk factors were documented as follows

a) Arterial hypertension: Blood Pressure e"140/90 mmHg before or one week after the stroke in supine posture. ${ }^{4}$

b) Diabetes mellitus: Documented by previous records before stroke event or a fasting plasma glucose e" $126 \mathrm{mg} \%$ or post prandial plasma glucose e" $200 \mathrm{mg} \%$ after seven days of acute event in two separate occasions. ${ }^{4}$

c) Hypercholesterolemia: Fasting blood cholesterol (8 hours of fasting) $>250 \mathrm{mg} \%$ after 7 days of acute event. ${ }^{4}$

d) Hyper triglyceridemia: Fasting triglyceride level $>150 \mathrm{mg} \%$ after $\&$ days of stroke. ${ }^{4}$

e) LDL Cholesterol: Total cholesterol- HDL Cholesterol- ${ }^{1} / 5$ Triglyceride. A value $>160 \mathrm{mg} \%$ is considered high. ${ }^{4}$

f) HDL Cholesterol: A value $<40 \mathrm{mg} \%$ is considered low. ${ }^{4}$

g) Smoking: This was defined as intake of more than 10 bidi or cigarette per day at the time of stroke. ${ }^{4}$

h) Lipoprotein(a): Fasting lipoprotein (a) level $>30 \mathrm{mg} \%$ considered high. ${ }^{5}$

i) Homocysteine : More than 10.8 micromole/litre is considered as high. ${ }^{5}$

j) hsCRP: Level of hsCRP $>1 \mathrm{mg} /$ litre are indicative of risk. ${ }^{6}$

k) Atrial Fibrillation: As per pulse and ECG. ${ }^{5}$

1) Left Vent Hypertrophy: By ECG and Echocardiography. ${ }^{5}$

m) Valvular heart diseases: By Echocardiographic examination.

n) H.pylori infection: As indicated by positive RUT with sample taken by upper GI Endoscopy. ${ }^{7}$

o) Carotid Intima Media Thickness: By carotid artery Doppler study-atherosclerotic plaque demonstrated by a thickness $>1.5 \mathrm{~mm}$ as measured from the mediaadventitia interface to the intima-lumen interface. ${ }^{8}$

\section{Result and Analysis:}

In this study 19 of the 30 patients were in the age bracket of 51 to 70 years. Mean age of the patients were 62.3 years. Male and female ratio in this study was 2:1. Presenting features of all the 30 patients at the time of admission was shown in Table-I.

Table-I

Presenting features at the time of admission

\begin{tabular}{lc}
\hline Presenting features & Number of patients \\
\hline Hemiparesis & 30 (Right-17 \& Left-13) \\
Slurring of speech & 20 \\
Deviation of angle of mouth & 18 \\
Altered level of consciousness & 9 \\
Ataxia & 2 \\
Bladder-bowel involvement & 17 \\
\hline
\end{tabular}

In table-II correlation between diabetes mellitus, blood $\mathrm{HbA} 1 \mathrm{C}$,hypertension,smoking with ischaemic stroke was 
shown. Among 30 patients, 9 were non diabetic, 5 were pre diabetic and 16 were diabetic. In our study correlation between diabetes mellitus and ischemic stroke was significant. ( chi square 7.177( $\mathrm{p}=0.007)$ ). Correlation between $\mathrm{Hb} \mathrm{A} 1 \mathrm{C}$ and ischemic stroke was also significant (chi square $4.022(p=0.045)) .25$ hypertensive patients were found within 30 patients. In our study correlation between hypertension and ischemic stroke was significant. (chi square 10 ( $\mathrm{p}=$ 0.000)). Among 30 patients, 15 were smoker. In our study correlation between smoking and ischemic stroke was significant. (chi square $4.593(\mathrm{p}=0.032)$ ). (Table-II)

In table-II correlation between lipid profile, Lp(a), hsCRP, homocysteine with ischaemic stroke was demonstrated.
Within 30 patients, abnormal lipid profile was observed in 18 cases. In our study correlation between dyslipidemia and ischemic stroke was significant. (chi square 6.787 ( $\mathrm{p}=$ 0.009). Abnormal lipoprotein(a) was observed in 23 cases. Correlation between lipoprotein(a) and ischemic stroke was significant.(chi square $15.017(p=0.000)$ ). Among 30 patients, abnormal hsCRP was seen in 17 cases. Correlation between hsCRP and ischemic stroke was significant. (chi square $10.335(\mathrm{p}=0.001)$ ). Among 30 patients, Abnormal homocysteine was observed in 22 cases out of the 30 patients. Correlation between hsCRP and ischemic stroke was significant. (chi square 17.143 $(p=0.000)) .($ Table-III $)$

Table-II

Correlation between Diabetes, HbA1C, Hypertension ,Smoking with ischaemic stroke

\begin{tabular}{lccccc}
\hline Diabetes & No in Cases & Control & Total & OR & CHISQUARE \\
\hline Yes & 16 & 6 & 22 & 4.577 & $7.177(\mathrm{p}=0.007)$ \\
No & 14 & 24 & 38 & & SIGNIFICANT \\
Total & 30 & 30 & 60 & OR & CHI SQUARE \\
Hb A1C & No in Cases & Control & Total & 3.333 & S.022(p=0.045) \\
$>6.5 \%$ & 12 & 5 & 17 & & SIGNIFICANT \\
d" $6.5 \%$ & 18 & 25 & 43 & & CHI SQUARE \\
Total & 30 & 30 & 60 & 10 & OR $(\mathrm{p}=0.000)$ \\
Hypertension & No in Cases & Control & Total & SIGNIFICANT \\
Yes & 25 & 10 & 35 & & CHI SQUARE \\
No & 5 & 20 & 25 & OR & 4.593(p=0.032) \\
Total & 30 & 30 & 60 & 3.286 & SIGNIFICANT \\
Smoking & No in Cases & Control & 7 & 22 & \\
Yes & 15 & 23 & 38 & 60 & \\
No & 15 & 30 & & & \\
Total & 30 & & & & \\
\hline
\end{tabular}

Table-III

Correlation between lipid profile, $L p(a)$, hsCRP, serum homocysteine with ischaemic stroke

\begin{tabular}{|c|c|c|c|c|c|}
\hline Lipid Profile & No in Cases & Control & Total & OR & CHI SQUARE \\
\hline Abnormal & 18 & 8 & 26 & 4.125 & $6.787(p=0.009)$ \\
\hline Normal & 12 & 22 & 34 & & SIGNIFICANT \\
\hline Total & 30 & 30 & 60 & & \\
\hline $\mathrm{LP}(\mathrm{a})$ & No in Cases & Control & Total & OR & CHISQUARE \\
\hline Increased & 23 & 8 & 31 & 9.036 & $15.017(\mathrm{p}=0.000)$ \\
\hline Normal & 7 & 22 & 29 & & SIGNIFICANT \\
\hline Total & 30 & 30 & 60 & & \\
\hline hsCRP & No in Cases & Control & Total & $\mathrm{OR}$ & CHI SQUARE \\
\hline Increased & 17 & 5 & 22 & 6.538 & $10.335(\mathrm{p}=0.001)$ \\
\hline Normal & 13 & 25 & 38 & & SIGNIFICANT \\
\hline Total & 30 & 30 & 60 & & \\
\hline \multicolumn{2}{|c|}{ Homocysteine levelNo in Cases } & Control & Total & OR & CHI SQUARE \\
\hline Increased & 22 & 6 & 28 & 11.00 & $17.143(\mathrm{p}=0.000)$ \\
\hline Normal & 8 & 24 & 32 & & SIGNIFICANT \\
\hline Total & 30 & 30 & 60 & & \\
\hline
\end{tabular}


Table-IV revealed correlation between different cardiac parameters with ischaemic stroke. Among 30 patients, abnormal LVH was observed in 15 cases. Our study revealed significant correlation between LVH and ischemic stroke .(chi square 5.934( $\mathrm{p}=0.015)$ ). Among 30 patients, abnormal CIMT was observed in 16 cases. significant correlation between CIMT and ischemic stroke was found in our study (chi square 5.711 $(\mathrm{p}=0.017))$. Valvular heart disease was observed in 3 cases. In our study correlation between valvular heart disease and ischemic stroke was not significant. (chi square $11.071(\mathrm{p}=0.612))$. Among 30 patients, AF was observed in 4 cases. Correlation between $\mathrm{AF}$ and ischemic stroke was not significant. (chi square $1.964(\mathrm{p}=0.161)$ ). (Table-IV)

Table V demonstrated relationship between H.pylori infection, ANA with ischaemic stroke. Among 30 patients, H.pylori infection was observed in 24 cases. Correlation between H.pylori infection and ischemic stroke was significant. (chi square 4.800 ( $\mathrm{p}=0.028)$ ). Among 30 patients, ANA was positive in 1 case. Correlation between positive ANA and ischemic stroke was not significant. (chi square 0 $(\mathrm{p}=1.000))$. (Table-V)

Table-IV

Correlation of different cardiac parameters with ischaemic stroke

\begin{tabular}{lccccc}
\hline LVH & No in Cases & Control & Total & OR & CHI SQUARE \\
\hline Present & 15 & 6 & 21 & 4 & $5.934(\mathrm{p}=0.015)$ \\
Absent & 15 & 24 & 39 & & SIGNIFICANT \\
Total & 30 & 30 & 60 & OR & CHI SQUARE \\
CIMT & No in Cases & Control & Total & 3.755 & $5.711(\mathrm{p}=0.017)$ \\
e"lmm & 16 & 7 & 23 & & SIGNIFICANT \\
$<1 \mathrm{~mm}$ & 14 & 23 & 37 & & \\
Total & 30 & 30 & 60 & OR & CHI SQUARE \\
Valvular heart diseaseNo in Cases & Control & Total & 3.22 & $11.071(\mathrm{p}=0.612)$ \\
Present & 3 & 1 & 4 & & \\
Absent & 27 & 29 & 56 & & \\
Total & 30 & 30 & 60 & OR & CHI SQUARE \\
AF & No in Cases & Control & Total & 4.462 & $1.964(\mathrm{p}=0.161)$ \\
Present & 4 & 1 & 5 & & \\
Absent & 26 & 29 & 55 & & \\
Total & 30 & 30 & 60 & & \\
\hline
\end{tabular}

Table-V

Correlation with H.Pylori infection and A.N.A with ischaemic stroke

\begin{tabular}{lccccc}
\hline H.pylori infectionNo in Cases & Control & Total & OR & CHI SQUARE \\
\hline Present & 24 & 16 & 40 & 3.500 & $4.800(\mathrm{p}=0.028)$ \\
Absent & 6 & 14 & 20 & & SIGNIFICANT \\
Total & 30 & 30 & 60 & OR & CHI SQUARE \\
ANA & No in Cases & Control & Total & 1 & $0(\mathrm{p}=1.000)$ \\
Present & 1 & 1 & 2 & & \\
Absent & 29 & 29 & 58 & 60 & \\
\hline Total & 30 & 30 & &
\end{tabular}




\section{Discussion:}

We have studied well documented different risk factors for ischemic stroke. So far hypertension, smoking, diabetes and dyslipidaemia have been studied extensively and different preventive strategies are being taken accordingly. However other novel risks factors which are less well documented but potentially modifiable are now emerging and thereby offer some insight for development of so called 'cryptogenic strokes'.

In this study male patients were outnumbered the female where $66.6 \%$ of study group were male. This is comparable with other studies from India by Nayak et al and Md Abu Naser Siddique et al from Bangladesh where more than 70\% male stroke patients have been documented. ${ }^{4}{ }^{9}$ Patricia $\mathrm{H}$ et al have shown that relative risk for stroke was 2.0 in case of male. ${ }^{10}$ In developing countries including ours it may be due to a referral bias.

In our study stroke was found most commonly in 51 to 70 years age group ( $63.33 \%$ of study population) and this is similar to studies from South East Asia and Western literature. ${ }^{9,11}$

In our study the frequency of diabetes mellitus, hypertension, smoking and dyslipidaemias are similar to the other studies of the world. We have found $53.33 \%$ patients were diabetic, $83.33 \%$ patients were hypertensive, $50 \%$ were smoker and $60 \%$ of patients were suffering from dyslipidaemias. These findings are in accordance to the similar studies done by Patricia $\mathrm{H}$ et al. The Dubbo Study and in ARIC Study. ${ }^{10,12,13}$ Another finding in our study was 12 cases $(40 \%)$ and 5 controls $(16.67 \%)$ had $\mathrm{HbA1C}>6.50 \%$. Smoking is another risk factor for ischemic stroke. ${ }^{11}$ When none of our female cases were smoker 15 of the 20 male cases were used to do. Similarly dyslipidaemia also found as very important risk factor in our study and it also confirms the finding of Anglo-Scandinavian Cardiac outcome Trial and The Veterans Administration HDL Intervention Trial. ${ }^{14}$ Tziomalos K et al showed elevated LDL-C levels appear to increase to increase the risk of stroke. Low HDL-C levels also appear to be associated with a greater risk whereas the importance of high triglyceride level is less clear. ${ }^{15} 18$ of our 30 cases showed some form of lipid abnormality.

There is evidence that Lipoprotein(a), Lp (a), is a predictor of many forms of vascular diseases, including premature coronary artery disease. Several studies have also evaluated the association between LP(a) and thrombotic stroke. In our study serum $L p(a)$ was raised in $73.33 \%$ cases and $20 \%$ of controls. It was high more in male than female. Smolders B et al showed Lp(a) was more frequently abnormally elevated among stroke patients( OR 2.39; 95\% CI 1.57 to 3.63). ${ }^{16,18}$ We also support their observation.

Homocystine is postulated to cause ischaemic stroke via various mechanisms. It may promote arherogenesis by damaging the vascular matrix, increasing oxidative injury to arterial endothelium and enhancing proliferation of vascular smooth muscle. High levels of Homocysteine have been associated with extra cranial carotid disease. It may also be prothrombotic and impair vasomotor regulation. Homocysteine is thus a biologically plausible factor in the pathogenesis of ischemic stroke in particular large artery strokes.

In our study $73.33 \%$ of patients who had high Homocysteine level suffered from infraction. Raised Homocysteine level was seen in $20 \%$ of controls. Raised level was found more in advanced age group. This correlation is explained on the basis of nutritional status and diminished vitamin intake which deteriorate with advance age. This observation is absolutely in accordance to the similar findings of Clarke $\mathrm{R}$ et al. ${ }^{17}$ Dhamija et al had documented raised homocysteine level to be independently associated with ischemic stroke and they also showed a positive correlation of plasma Homocysteine with smoking. ${ }^{18}$ But we have not found any such relationship with smoking. So it can be concluded that relationship with Homocysteine and stroke risk is strong, graded and significant.

Regarding hsCRP 17 cases (56.67\%) and 5 controls (16.67\%) had elevated levels. Thus a positive correlation was documented and this also in accordance to the study done by Paul M Ridker et al. ${ }^{6}$ In our study 24 cases ( $80 \%$ ) and 16 controls $(20 \%)$ had H. pylori infection. There was significant correlation between $\mathrm{H}$. pylori infection and ischemic stroke as per as our study. Same observation has been documented by Hugh $\mathrm{S}$ et al as they have noted the odd ratio for cerebrovascular disease associated with $\mathrm{H}$. pylori seropositivity was 1.78 ( $95 \%$ confidence interval $(95 \% \mathrm{CI}$ ) 1.14-2.77) and this remain significant after controlling for other risk factors including socioeconomic status $(1.63$ (95\% CI 1.02-2.60). ${ }^{19}$ Peter U Heuschmann et al have found similar association between $\mathrm{H}$. pylori infection and Ischemic stroke by observing high rate of small artery occlusion ( adjusted odds ratio, $3.31 ; 95 \%$ CI, 1.15 to 9.56 ) and a lower risk of cardio embolic stroke (adjusted odds ratio, $0.21 ; 95 \%$ CI, 0.06 to 0.71$).{ }^{7}$ Regarding Carotid Doppler study 16 cases $(53.33 \%)$ and 7 controls $(23.33 \%)$ had atheromatous plaque in their carotid artery. The Framingham study reported that age, cigarette smoking, diabetes, systolic blood pressure and high cholesterol were independent risk factors of carotid atherosclerosis. In the Rotterdam study, Michiel L. Bots et al 
showed ischemic stroke risk increased gradually with increasing IMT. The odds ratio for stroke per standard deviation increase $(0.163 \mathrm{~mm})$ was $1.41(95 \% \mathrm{CI}, 1.25$ to 1.82$){ }^{20}$

In our study 15 cases $(50 \%)$ and 6 controls(20\%) had left ventricular hypertrophy (LVH) and it was significantly associated with ischaemic stroke. We have also noticed that LVH was strongly associated with hypertension. Eurostroke projects showed LVH was associated with twofold increased risk of stroke (odd ratio2.1 (95\% CI 1.3 to 3.5 ). ${ }^{21}$ The Framingham study showed atrial fibrillation is an independent risk factor for stroke. ${ }^{22}$ All cardiac emboli strokes in our study were due to mitral stenosis complicated with atrial fibrillation (13.33\% of the patients). We have not noted any significant association either with atrial fibrillation or with any valvular heart disease and ischaemic stroke. These are in contrary to most studies. However our small number of patients may not have reflected the true picture. Anti-Nuclear Antibody(ANA) was positive only in 3.33\% of our patients. As the sample size was small and as we had not performed intra cerebral angiography for detection of vasculitis, it is difficult to conclude anything regarding role of SLE in ischaemic stroke.

\section{Summary and Conclusion:}

In our study there were 30 cases of ischaemic stroke of which $20(67.66 \%)$ were male. Among them only $1(3.33 \%)$ was within age group 21 to 30 years and $19(63.33 \%)$ patients were in the age group of 51 to 70 years.

In our study 16 patients $(53 \%)$ were diabetic which is statistically significant (p value 0.007 ) and 25 patients $(83 \%)$ were hypertensive which is also statistically significant ( $p$ value 0.000$) .15$ of the 30 case $(50 \%)$ were smoker and it is also statistically significant ( $\mathrm{p}$ value 0.032 ). Dyslipidaemia, another stroke risk factor for stroke was found in $18(60 \%)$ of cases which indicate a positive correlation having a $\mathrm{p}$ value 0.009 . High homocysteine level was found in 22 case $(73.33 \%)$ and here also correlation between homocysteine level and ischaemic stroke is significant ( $p=0.000$ ). Similarly high $\mathrm{Lp}(\mathrm{a})$ level is significantly associated with ischaemic stroke as 23 cases $(73.33 \%)$ showed high level $(p=0.000)$. Elevated homocysteine level was associated with elevated Lp (a) level in 17 cases. Statistically significant correlation ( $p$ value 0.001 ) between stroke and high hsCRP has been observed in this study as 17 of the 30 cases $(56.67 \%)$ had high levels. LVH was evident in 15 cases $(50 \%)$ of stroke patients having a statistically significant correlation ( $\mathrm{p}$ value 0.015 ). Among the other findings atheromatous plaque was documented in 16 cases $(53.33 \%)$ and this also has a statistically significant (P value 0.017) risk factor for development of stroke. Another risk factor documentation was associated $\mathrm{H}$. pylori infection in stroke when 24 cases $(80 \%)$ had positive results and statistically the $\mathrm{p}$ value is 0.028 . We failed to document any significant association between stroke and ANA positivity as only 1 case $(3.33 \%)$ was found positive similar to that found in control group ( $p$ value 1.000). Same words are applicable for AF and Valvular heart diseases as only 4 cases (13.33\%) had these problems which is statistically insignificant ( $p$ value 0.161 -Fisher' exact test) for risk factors for stroke. These findings need further confirmation because of our sample size and control was small. IFrom this study this can be concluded that like other parts of the world, hypertension, diabetes mellitus, smoking, dyslipidaemia are equally important risk factors for ischemic stroke in our country. Moreover elevated lipoprotein(a), hyperhomocysteinemia, inflammation, H. pylori infection which are much discussed newer risk association of stroke is also prevalent in this part of the world.

We recommend that in addition to measures that include blood sugar, blood pressure and lipid control routine estimation of $\mathrm{Lp}(\mathrm{a})$ and homocysteine level should be done so that appropriate steps can be taken to avert an attack of stroke. Similarly in high risk subjects monitoring of atherosclerotic changes in carotid artery by Doppler study and Echocardiographic monitoring of LVH should be done to alert the subject about stroke. Lastly early detection and eradication of H.pylori is always advisable to reduce the incidence of ischemic stroke.

\section{Acknowledgement: None}

\section{Conflict of Interest : None}

\section{References:}

1. Sanchetee P.Stroke in young. JIACM 2002;3:228-30.

2. Rosamond W, Flegal K, Friday G, Furie $K$, Go A, Greenlund $K$, et al .Heart disease and stroke statistics-2007 update: a report from the American Heart Association Statistics Committee and Stroke Statistics Subcommittee. Circulation $2007 ; 115$.

3. World Health Organization (1978). Cerebrovascular Disorders (offset Publication) Geneva: World Health Organization . ISBN9241700432.OCLC4757533

4. Nayak SD, Nair,M, Radhakrishnan K, Sarma PS. Ischaemic stroke in the young adult: Clinical features, risk factors and outcome. Natl Med J India 1997;10:107-12.

5. Goldstein LB, Adams R, Alberts MJ, Appel LJ, Brass LM, Bushnell CD, Primary prevention of ischemic stroke. Stroke. 2006;37:1583-633.

6. Ridker PM, Rifai N, Rose L, Buring JE, Cook NR. Comparison of C-reactive protein and low-density lipoprotein cholesterol levels in the prediction of first cardiovascular events. N Engl J Med 2002;347:1557-65 
7. Heuschmann PU, Neureiter D, Gesslein M, Craiovan B, Maass M, Faller G, et al. Association between infection with helicobacter pylori and chlamydia pneumoniae and risk of ischemic stroke subtypes. [Results from a PopulationBased Case-Control Study]. Stroke. 2001; 32: 2253-58.

8. Touboul PJ, Hennerici MG, Meairs S, Adams H, Amarenco $\mathrm{P}$, Bornstein N, et al. Mannheim carotid intima-media thickness consensus (2004-2006). An update on behalf of the Advisory Board of the 3rd and 4th Watching the Risk Symposium, 13th and 15th European Stroke Conferences, Mannheim, Germany, 2004, and Brussels, Belgium, 2006. Cerebrovasc Dis. 2007;23:75-80.

9. Siddique MAN, Nur Z, Mahbub M.S, Alam M.B, Miah T.M. Clinical presentation and epidemiology of stroke: A study of 100 cases. Journal of Medicine 2007;10:86-89.

10. Patricia H . Risk factors for ischemic stroke: A prospective study in Rochester, Minnesota. Annals of Neurology 1987;22:319-27.

11. Whisnant JP .Modeling of risk factors for ischemic stroke: The Willis Lecture. Stroke 1997;28:1840-44.

12. Simons LA, McCallum J, Friedlander Y, Simons J et al. Risk Factors for Ischemic Stroke: Dubbo Study of the Elderly. Stroke 1998;29:1341-46.

13. Chambless LE, Folsom AR, Clegg LX, Sharrett AR, Shahar E, Nieto FJ. et al.Carotid wall thickness is predictive of incident clinical stroke: the Atherosclerosis Risk in Communities (ARIC) study. Am J Epidemiol. 2000; 151 :478-87.

14. Bloomfield Rubins H, Davenport J, Babikian V, Brass LM, Collins D, Wexler L et al. Reduction in Stroke With Gemfibrozil in Men With Coronary Heart Disease and Low
HDL Cholesterol :The Veterans Affairs HDL Intervention Trial (VA-HIT). Circulation. 2001;103:2828-33.

15. Tziomalos K, Athyros VG, Karagiannis A, Mikhailidis DP. Dyslipidemia as a risk factor for ischemic stroke. Curr Top Med Chem. 2009;9:1291-7.

16. Smolders B, Lemmens R, Thijs V.Lipoprotein (a) and Stroke: A Meta-Analysis of Observational Studies. Stroke. 2007; 38: 1959-66.

17. Clarke R, Halsey J, Lewington S, Lonn E, Armitage J,Manson $\mathrm{JE}$ et al. Effects of lowering homocysteine levels with B vitamins on cardiovascular disease, cancer, and cause-specific mortality: Meta-analysis of 8 randomized trials involving 37,485 individuals. Arch Intern Med. 2010;170:1622-31.

18. Dhamija KR, , Gaba P, Arora S., Kaintura A, Kumar M, Bhattacharjee J. Homocysteine and lipoprotein (a) correlation in ischemic stroke patients. J Neurol Sci 2009;281:64-8.

19. Hugh SM, Mendall M.A. Helicobacter pylori infection: a risk factor for ischaemic cerebrovascular disease and carotid atheroma. J Neurol Neurosurg Psychiatry 1998;64:104-7.

20. Bots ML, Hoes AW, Koudstaal PJ, Hofman A, Grobbee DE. Common Carotid Intima-Media Thickness and Risk of Stroke and Myocardial Infarction. Circulation. 1997; 96:1432-37.

21. Bots ML, Nikitin Y, Salonen JT, Elwood PC, Malyutina S, Freire de Concalves A, et al. Left ventricular hypertrophy and risk of fatal and non-fatal stroke. EUROSTROKE: a collaborative study among research centres in Europe. J Epidemiol Community Health 2002; 56:i8-i13.

22. Wolf PA Abbott RD, Kannel WB. Atrial fibrillation as an independent risk factor for stroke: the Framingham Study. Stroke.1991; 22: 983-8. 